\title{
Primary Care Asssement Tool (PCAT): a construção de uma nova linha de base para avaliação dos serviços de saúde no Brasil
}

\author{
Primary Care Assessment Tool (PCAT): developing a new baseline \\ for evaluating Brazilian health services
}

Luiz Felipe Pinto (https://orcid.org/0000-0002-9888-606X) ${ }^{1}$

Vinicius Siqueira Tavares Meira Silva (https://orcid.org/0000-0002-3271-0811) 1,2

${ }^{1}$ Programa de PósGraduação em Atenção Primária à Saúde, Faculdade de Medicina, Universidade Federal do Rio de Janeiro. Av. Presidente Vargas 2863, Cidade Nova. 20210-030 Rio de Janeiro RJ Brasil. felipepinto.rio@ medicina.ufrj.br ${ }^{2}$ Programa de Residência em Medicina de Família e Comunidade, Secretaria Municipal de Saúde do Rio de Janeiro. Rio de Janeiro RJ Brasil.

\begin{abstract}
In Brazil, within the SUS, Primary Health Care (PHC) gained relevance from the Family Health Strategy's structuring from the 1990s to the 2000s. Several instruments are available in the world to evaluate PHC services, including the family of instruments of the Primary Care Assessment Tool (PCAT), developed and disseminated by Starfield \& Shi to assess the existence and extent of the features of primary health care services. Reinforcing the importance of using this instrument in Brazil, the Ministry of Health published in 2020 a new edition of the Brazilian version that informs the methodology used for such instruments, reviving the role of IBGE as a significant external evaluator of the SUS. The IBGE pioneered in its primary household random sample survey, the National Health Survey, a question-based module of the reduced version of the PCAT for adult users. The leading global results found for Brazil (overall PCAT score=5.9) inform that those who use PHC services (adults with referred morbidities) the most are also those who evaluate these services most positively. Differences were also observed among the residents of households registered by the family health teams, those receiving visits from the community and endemic workers, and age groups (older people evaluate services more positively).
\end{abstract}

Key words Primary Health Care, Health evaluation, Household surveys, PCAT, Brazil
Resumo No Brasil, no âmbito do SUS, a APS ganhou relevância a partir da estruturação da Estratégia Saúde da Família entre as décadas de 1990/2000. Existem inumeros instrumentos para a avaliação desses serviços no mundo. Dentre eles, destaca-se a família de instrumentos do Primary Care Assessment Tool (PCAT), desenvolvida e disseminada por Starfield e Shi para avaliar a existência e extensão das características dos serviços de atenção primária em saúde. Reforçando a importância do uso desse instrumento no Brasil, o Ministério da Saúde publicou em 2020, uma nova edição da versão brasileira que informa a metodologia utilizada, resgatando o papel do IBGE como grande avaliador externo do SUS. O IBGE incluiu de forma pioneira em seu principal inquérito amostral domiciliar, a Pesquisa Nacional de Saúde, um módulo de perguntas da versão reduzida do PCAT para usuários adultos. Os principais resultados globais encontrados (escore geral $=5,9$ ) informam que aqueles que mais utilizam os serviços de APS (adultos com morbidades referidas), são também os que avaliam mais positivamente tais serviços. Também foram observadas diferenças entre os moradores de domicílios cadastrados pelas equipes de Saúde da Família, entre os que recebem visitas de agentes comunitários e agentes de endemias e, por faixa etária (os mais idosos avaliam de forma mais positiva os serviços).

Palavras-chave Atenção Primária à Saúde, Avaliação em saúde, Inquéritos domiciliares, PCAT, Brasil 


\section{Introdução}

A Declaração de Alma-Ata $(1978)^{1}$ marca uma mudança no paradigma da saúde global ao instituir a Atenção Primária à Saúde (APS) como um elemento essencial para a garantia da saúde como um direito humano universal. A APS compreende o primeiro nível de cuidado ao usuário, funcionando como sua porta de entrada ao sistema de saúde, e tem ocupado uma posição cada vez mais forte e central na organização dos sistemas nacionais em países como o Reino Unido, Portugal, Espanha, Canadá, dentre outros ${ }^{2-4}$.

No Brasil, no âmbito do Sistema Único de Saúde (SUS), a APS ganhou relevância a partir da estruturação da Estratégia Saúde da Família (ESF) entre as décadas de 1990 e 2000, um modelo de organização deste nível de atenção com características peculiares, como o trabalho em equipes multiprofissionais e orientação comunitária - os agentes comunitários de saúde - reforçando o vínculo com o território.

Existem inúmeros instrumentos para a avaliação dos serviços de APS no mundo. O Primary Care Assessment Tool (PCAT) foi desenvolvido e disseminado por Starfield e Shi ${ }^{5-8}$ buscando traçar a orientação dos serviços para os chamados "atributos essenciais" propostos para a APS (acesso de primeiro contato, longitudinalidade, integralidade e coordenação do cuidado), além de três dimensões classificadas como "derivadas" (orientação familiar e comunitária e competência cultural $)^{9}$.

O PCAT possui algumas versões-espelho de seus questionários. Por isso, usualmente falamos de "famílias de instrumentos PCAT", conforme o público-alvo (crianças, adultos, profissionais de saúde - médicos, enfermeiros, cirurgiões-dentista - , gerentes/gestores). Foi traduzido, validado estatisticamente e vem sendo utilizado em sua totalidade ou em parte, por dezenas de países em todos os cinco continentes do mundo ${ }^{10}$. Algumas versões reduzidas para adultos foram propostas e validadas, com o intuito de permitir maior praticidade, redução de custos na aplicação e viabilidade técnica no "mundo real" da gestão em saúde. Nessa direção, o Instituto Brasileiro de Geografia e Estatística (IBGE) inovou ao incluir na última PNS-2019 a versão curta com 25 itens do referido instrumento para usuários adultos ${ }^{11,12}$.

O IBGE é a instituição governamental responsável pela produção de informações sobre a população brasileira. Ele coordena desde 2013, a Pesquisa Nacional de Saúde (PNS), um inquérito domiciliar de base populacional cuja última versão foi a campo a partir de agosto de 2019. A PNS-2019 alcançou todas as 27 unidades da federação e inquiriu a população acerca de uma extensa variedade de temas relacionados às características sociodemográficas, condições de saúde, hábitos e estilos de vida, questões relacionadas à utilização de serviços de saúde, além de obter medidas antropométricas de uma subamostra.

\section{Sobre o material e os métodos}

A PNS-2019 é uma pesquisa desenvolvida com amostragem probabilística por conglomerados em três estágios. No primeiro estágio foram selecionadas 8.036 unidades primárias de amostragem (UPA) por amostragem aleatória simples. No segundo, também por amostragem aleatória simples, foram determinados os domicílios visitados, variando em quantidade, entre 12 e 18 domicílios por UPA, conforme o tamanho de cada um dos 27 estados da federação. No terceiro, todos moradores do domicílio são entrevistados ou, em alguns Módulos do instrumento, como o de atenção primária à saúde, um morador adulto com 18 anos ou mais, é aleatoriamente selecionado para responder.

$\mathrm{O}$ inquérito incorporou, pela primeira vez, a versão curta validada do PCAT para adultos. Contidas no "Módulo H", essas questões foram aplicadas a indivíduos com 18 anos ou mais que afirmaram ter procurado um atendimento médico em uma unidade de atenção primária à saúde (com ou sem Equipe de Saúde da Família (eSF)) nos seis meses anteriores e que também já haviam sido atendidos pelo mesmo profissional previamente pelo menos uma vez, resultando em uma população de 17,2 milhões de pessoas (após a expansão da amostra realizada de cerca de 10.000 respondentes).

Os dados são obtidos por meio da aplicação de um questionário em escala Likert de quatro pontos. Atribui-se um valor numérico para cada resposta (de 1 a 4 , do menos positivo ao mais positivo), que é a base para o cálculo da média de todas as respostas. Nas versões do instrumento publicadas pelo Ministério da Saúde em 2010 e, atualizada em $2020^{10,13}$ essas respostas numéricas são, posteriormente, transformadas em uma nota (escore) com variação de 0 a 10 . Dessa forma, a interpretação dos resultados fica facilitada por usar uma escala numérica muito comum no dia a dia da gestão em saúde que é atribuir uma nota nessa escala para avaliar uma ação, programa ou serviço de saúde. Este escore indica a maior ou 
menor presença e extensão dos atributos da APS no serviço sob avaliação, podendo ser classificado em "alto" quando maior ou igual a 6,6 e "baixo" se menor que 6,6. Dito de uma outra forma, se for obtido $2 / 3$ da nota em cada quesito, atributo ou escore, diz-se que aquele atributo ou conjunto de serviços está adequadamente orientado à APS.

\section{O processo de coleta de dados no contexto brasileiro em suas dimensões continentais}

O Brasil é um país de características continentais. Sua vasta extensão territorial impõe desafios logísticos para a realização de pesquisas domiciliares de alcance nacional, como a PNS. De um lado, há enorme concentração populacional em metrópoles densamente povoadas e, de outro, regiões como a área interestadual da Amazônia Legal, com baixa ocupação, difícil acesso e infraestrutura de transporte precárias e limitadas. O processo de coleta de dados na PNS-2019 pode ser considerado como o maior esforço demográfico de registro de dados de saúde de base domiciliar, depois dos Censos decenais.

\section{Resultados do Primary Care Assessment Tool (PCAT) na PNS-2019}

Os primeiros resultados divulgados pelo IBGE reportam-se à comparação do escore geral obtido no questionário segundo algumas variáveis sociodemográficas: (1) sexo, (2) faixa etária, (3) raça/cor, (4) faixas de rendimento domiciliar per capita, (5) estado conjugal, (6) comorbidades selecionadas: hipertensão arterial, diabetes, doença do coração, asma, depressão, doença crônica no pulmão. Também são apresentados os escores conforme características do domicílio relacionadas à oferta de serviços da APS, tais como seu cadastro em uma unidade de saúde com Equipes de Saúde da Família, e a realização de visitas domiciliares pelos agentes comunitários de saúde (ACS) e de combate a endemias (ACE) nos últimos 12 meses (Tabela 1).

A PNS-2019 apresentou um escore geral para a APS no Brasil de 5,9, ou seja, abaixo do valor de referência de 6,6 preconizado na metodologia do instrumento utilizado para avaliação dos serviços. Contudo, quando os resultados são estratificados por características sociodemográficas ou presença de morbidades relevantes para a APS, são encontradas variações. As mulheres utilizaram mais o serviço de APS do que os homens. Porém, a sua avaliação foi semelhante em ambos os sexos, gerando um escore geral de 5,9 e 5,8 para mulheres e homens, respectivamente. Mas, quando observados os resultados por faixa etária, houve variação. Os mais velhos, a partir dos 60 anos de idade, avaliaram melhor os serviços do que os mais jovens, havendo uma elevação progressiva do escore geral conforme o aumento da faixa etária. Os valores foram de 5,6 na faixa entre 18 e 39 anos, 5,9 entre 40 e 59 anos e 6,1 a partir dos 60 anos. Os resultados foram idênticos para as populações declaradas brancas e pretas/ pardas, ambas indicando um escore geral de 5,9 para a APS. A avaliação da APS também não variou segundo o estado conjugal, alcançando um escore geral de 5,9 em todos os grupos.

Outras diferenças importantes observadas para o Brasil, referem-se ao melhor desempenho dos serviços de atenção primária à saúde, quando se consideram os domicílios cadastrados vs não cadastrados em unidades de saúde com Equipes de Saúde da Família (eSF). No primeiro caso, o escore geral $=6,0[5,9-6,1]$ e no segundo, 5,5 $[5,4$ - 5,7], demonstrando, portanto, o acerto da Política Nacional de Atenção Básica nas últimas décadas na aposta pelo fortalecimento e expansão da Estratégia de Saúde da Família pelas regiões geográficas do país. Também, a visita de agentes comunitários ou outros membros das eSF trouxe estimativas mais favoráveis entre aqueles domicílios que receberam pelo menos uma visita nos últimos 12 meses (escore geral=6,1 [6,0 - 6,2] vs escore geral=5,7 $[5,5-5,8]$ entre os domicílios que nunca receberam visita).

As pessoas com renda familiar per capita de até um salário mínimo, no momento da entrevista, foram as que mais utilizaram os serviços de APS. Porém, a renda familiar per capita pareceu não exercer influência sobre a avaliação da APS, nesta pesquisa. $\mathrm{O}$ escore geral na faixa de renda familiar per capita de até um salário mínimo foi de 5,8; entre um e três salários mínimos foi de 6,0 e, acima de três salários mínimos foi de 5,8.

O estudo estima, com respectivos intervalos de confiança, a presença de uma lista de morbidades de interesse para a APS na população. De modo geral, a avaliação dos serviços de APS foi superior entre os entrevistados que relataram portar alguma dessas doenças, em relação aos que negaram. Nos indivíduos que declararam o diagnóstico de hipertensão arterial, o escore geral foi de 6,2, enquanto nos que negaram foi de 5,7, da mesma forma para diabetes $(6,3$ nos portadores e 5,8 nos não portadores), doenças do coração (6,4 nos portadores e 5,8 nos não portadores), asma (6,0 nos portadores e 5,9 nos não portado- 
Tabela 1. Escore médio geral da atenção primária à saúde (valor de 0 a 10) com indicação do intervalo de confiança. Brasil, 2019.

\begin{tabular}{|c|c|c|}
\hline Variáveis selecionadas & Escore geral & IC95\% \\
\hline \multicolumn{3}{|l|}{ Sexo } \\
\hline Homem & 5,9 & {$[5,8-6,0]$} \\
\hline Mulher & 5,8 & {$[5,8-5,9]$} \\
\hline \multicolumn{3}{|l|}{ Grupos de idade } \\
\hline 18 a 39 & 5,6 & {$[5,5-5,7]$} \\
\hline 40 a 59 & 5,9 & {$[5,7-6,0]$} \\
\hline 60 ou mais & 6,1 & {$[6,0-6,2]$} \\
\hline \multicolumn{3}{|l|}{ Cor ou raça } \\
\hline Branco & 5,9 & {$[5,7-6,0]$} \\
\hline Preto ou pardo & 5,9 & {$[5,8-6,0]$} \\
\hline \multicolumn{3}{|l|}{ Faixas de rendimento domiciliar per capita } \\
\hline Até 1 salário mínimo & 5,8 & {$[5,7-5,9]$} \\
\hline Mais de 1 a 3 salários mínimos & 6,0 & {$[5,9-6,2]$} \\
\hline Mais de 3 salários mínimos & 5,8 & {$[5,4-6,2]$} \\
\hline \multicolumn{3}{|l|}{ Estado conjugal } \\
\hline Tem cônjuge & 5,9 & {$[5,8-6,0]$} \\
\hline Não tem cônjuge & 5,9 & {$[5,8-6,0]$} \\
\hline \multicolumn{3}{|l|}{ Hipertensão arterial } \\
\hline Sim & 6,2 & {$[6,1-6,3]$} \\
\hline Não & 5,7 & {$[5,6-5,8]$} \\
\hline \multicolumn{3}{|l|}{ Diabetes } \\
\hline Sim & 6,3 & {$[6,1-6,4]$} \\
\hline Não & 5,8 & {$[5,7-5,9]$} \\
\hline \multicolumn{3}{|l|}{ Doença do coração } \\
\hline Sim & 6,4 & {$[6,1-6,6]$} \\
\hline Não & 5,8 & {$[5,7-5,9]$} \\
\hline \multicolumn{3}{|l|}{ Asma } \\
\hline Sim & 6,0 & {$[5,7-6,3]$} \\
\hline Não & 5,9 & {$[5,8-5,9]$} \\
\hline \multicolumn{3}{|l|}{ Depressão } \\
\hline Sim & 6,1 & {$[5,9-6,2]$} \\
\hline Não & 5,8 & {$[5,8-5,9]$} \\
\hline \multicolumn{3}{|l|}{ Doença crônica no pulmão } \\
\hline Sim & 6,4 & {$[6,0-6,8]$} \\
\hline Não & 5,9 & {$[5,8-5,9]$} \\
\hline \multicolumn{3}{|c|}{ Domicílio está cadastrado na unidade de saúde da família?* } \\
\hline Sim & 6,0 & {$[5,9-6,1]$} \\
\hline Não & 5,5 & {$[5,4-5,7]$} \\
\hline \multicolumn{3}{|l|}{ Recebeu visita de ACS nos últimos 12 meses? } \\
\hline Pelo menos uma vez & 6,1 & {$[6,0-6,2]$} \\
\hline Nunca recebeu & 5,7 & {$[5,5-5,8]$} \\
\hline \multicolumn{3}{|l|}{ Recebeu visita de ACE nos últimos 12 meses? } \\
\hline Pelo menos uma vez & 6,0 & {$[5,9-6,1]$} \\
\hline Nunca recebeu & 5,6 & {$[5,4-5,7]$} \\
\hline
\end{tabular}

Fonte: IBGE, Diretoria de Pesquisas, Coordenação de Trabalho e Rendimento, Pesquisa Nacional de Saúde 2019, resultados divulgados em outubro de 2020.

Notas: A tabela considera o seguinte universo, de acordo com a metodologia do PCAT na PNS-2019: pessoas com 18 anos ou mais que procuraram algum serviço da Atenção Primária de Saúde (posto de saúde, centro de saúde ou unidade com equipes de saúde da família) nos últimos seis meses anteriores à entrevista e este atendimento não foi o primeiro realizado com o mesmo médico no serviço. As perguntas sobre morbidades referidas foram feitas, arguindo o morador selecionado sobre se: "algum médico(a) já lhe deu o diagnóstico de...” ${ }^{\star}$ Exclusive as pessoas que não souberam informar se o domicílio estava cadastrado em unidade com equipes de Saúde da Família. 
res), depressão (6,1 nos portadores e 5,8 nos não portadores) e doenças crônicas do pulmão $(6,4$ nos portadores e 5,9 nos não portadores).

\section{Discussão}

As evidências trazidas pelo PCAT na PNS-2019 apontam no sentido favorável ao modelo de atenção primária brasileiro no SUS, ancorado nas equipes de saúde da família, que os usuários avaliaram de forma mais positiva, espelhando um escore médio geral superior àquele observado entre as pessoas-residentes em domicílios não cadastrados por essas equipes.

Os primeiros dados publicados pelo IBGE são agregados para o total do País, o que impede a identificação de diferenças regionais entre as unidades da federação (UF) na avaliação da APS. Análises pormenorizadas serão viabilizadas com a publicação dos resultados para as regiões geográficas, UF, regiões metropolitanas, municípios do interior e municípios das capitais. Como sabemos, em pesquisas sociais, indicadores agregados costumam esconder variabilidades internas, isto é, “a média Brasil” nem sempre representará de forma homogênea o conjunto das partes.

A incorporação do PCAT no questionário da PNS, uma pesquisa de alcance e representatividade estatística nacional, permite que seus resultados sejam comparados com a ampla produção científica que se apoia no mesmo método. Isto só é possível porque o PCAT é um instrumento padronizado e validado internacionalmente para a avaliação de serviços de APS. O próprio Ministério da Saúde passou a incorporar a possibilidade de uso dessa metodologia a partir da Portaria $n^{\circ}$ 3.222 de 10 de dezembro de $2019^{14}$ que definiu indicadores de pagamento por desempenho, como uma das partes de um sistema de avaliação mais amplo para os serviços de APS no Brasil ${ }^{15}$.

A adoção de novas tecnologias para coleta de dados de pesquisas amostrais com validade externa, como entrevistas telefônicas, pode ajudar a superar o desafio logístico dos inquéritos domiciliares no Brasil. Recentemente, o IBGE empregou com êxito esta metodologia na coleta de dados para uma versão especial da Pesquisa Nacional por Amostra de Domicílio Contínua (PNAD-C) sobre a pandemia de COVID-19 ${ }^{16}$. Recomendamos que o mesmo processo de coleta seja realizado para as futuras PNS, ou seja, que pelo menos os módulos iniciais e o módulo sobre atenção primária à saúde possam ser coletados trimestralmente e seguir o mesmo calendário da PNAD-C já consolidado com as divulgações das estatísticas de mercado de trabalho divulgadas pelo Instituto.

\section{À guisa da conclusão}

A incorporação do PCAT versão reduzida no questionário da PNS-2019 representou uma inovação histórica dos mais de 80 anos de existência do IBGE. Seus resultados são pioneiros entre os institutos oficiais de estatística no mundo e comparáveis a diversos estudos locais brasileiros e internacionais. As diversas versões do instrumento passam por um processo de validação que almeja garantir a estabilidade e perenidade de seu conteúdo.

Apesar da falta de clareza e consenso na literatura quanto à escolha de um instrumento de avaliação de serviços de saúde, as experiências do Brasil (PNS-2019) e da Catalunha (Encuesta de Salud de Barcelona 2016-2017) ${ }^{17}$ ao agregarem versões reduzidas do PCAT a seus inquéritos nacionais/regionais domiciliares foram exitosas: conseguem traçar uma linha de base para futuras comparações e estudos mais desagregados por regiões/locorregiões, estados e municípios. Devido a sua fácil operacionalização e tempo curto de aplicação junto a população, o desafio está lançado: conseguirá o Brasil, apesar de todas as dificuldades de acessibilidade geográfica, perenizar/atualizar o uso desse instrumento e, com isso, monitorar a principal política pública de saúde, base de todos os sistemas universais de saúde, que é a APS? Um caminho que tentamos aqui demonstrar é a proposta de inclusão definitiva dessa temática na agenda do IBGE em sua PNAD Contínua, resgatando o papel de grande avaliador externo do SUS e contribuindo para análise das desigualdades de acesso, utilização e percepção da população brasileira sobre os atributos da APS, utilizando-se metodologia científica sólida, independente e consolidada nos últimos vinte anos pelo mundo acadêmico.

Por tudo isso, é alvissareira a iniciativa do IBGE, no apagar das luzes de seu questionário na Pesquisa Nacional de Saúde (PNS-2019), de inclusão do módulo do PCAT, que trouxe uma nova linha de base para avaliação e análise comparada dos serviços de APS no Brasil, na perspectiva dos usuários do SUS. 


\section{Colaboradores}

LF Pinto e VSTM Silva participaram igualmente de todas as etapas de elaboração do artigo. LF Pinto revisou criticamente a última versão do mesmo.

\section{Referências}

1. Declaração de Alma Ata sobre Cuidados Primários [Internet]. 1978 [acessado 2020 Out 28]. Disponível em: http://bvsms.saude.gov.br/bvs/publicacoes/declaracao_alma_ata.pdf

2. Saltman RB, Rico A, Boerma WGW. Primary care in the driver's seat? Organizational reform in European primary care. Berkshire: Open University Press; 2006.

3. Pisco L. Reforma da Atenção Primária em Portugal em duplo movimento: unidades assistenciais autónomas de saúde familiar e gestão em agrupamentos de Centros de Saúde. Cien Saude Colet 2011; 16(6):28412852.

4. Ponka D, Pinto LF, Whalen-Browne M, Meuser A, Prado JC, Michaelides O, Rouleau K. Contrasting current challenges from the Brazilian and Canadian national health systems. The Besrour Papers: a series on the state of family medicine in Canada and Brazil. Canadian Family Physician December 2019, 65(12):890-896.

5. Starfield B, Cassady CE, Nanda J, Forrest CB, Berk R. Consumer experiences and provider perceptions of the quality of primary care: implications for managed care. J Fam Pract 1998; 46(3):216-226.

6. Cassady CE, Starfield B, Hurtado MP, Berk RA, Nanda JP, Friedenberg LA. Measuring consumer experiences with primary care. Pediatrics 2000; 105(4 Pt 2):9981003.

7. Shi L, Starfield B, Xu J. Validating the Adult Primary Care Assessment Tool. J Family Practice 2001; 50(2):161-175.

8. Starfield B. Atenção primária: Equilíbrio entre necessidades de saúde, serviços e tecnologia. Brasília: Unesco, MS; 2002.

9. Shi L. The impact of primary care: a focused review. Scientifica (Cairo) 2012; 1-22.

10. Brasil. Ministério da Saúde (MS). Secretaria de Atenção Primária à Saúde. Departamento de Saúde da Família. Manual do Instrumento de Avaliação da Atenção Primária à Saúde: PCATool-Brasil - 2020. Brasília: MS; 2020.

11. Harzheim E, Pinto LF, D'Ávila OP, Hauser L. Following the legacy of professors Barbara Starfield and Leiyu Shi in Brazil as health policy: the National Health Survey (PNS), led by the Brazilian National Institute of Geography and Statistics (IBGE) and the Primary Care Assessment Tool (PCAT). Int J Equity Health 2019; 18(1):176.
12. Instituto Brasileiro de Geografia e Estatística (IBGE). Pesquisa Nacional de Saúde. IBGE investiga pela primeira vez recepção dos pacientes no sistema público de saúde [Internet]. Agência de Notícias: IBGE; 2019 [acessado 2020 Nov 03]. Disponível em: https://agenciadenoticias.ibge.gov.br/ agencia-noticias/2012-agencia-de-noticias/noticias/25536-ibge-investiga-pela-primeira-vez-recepcao-dos-pacientes-no-sistema-publico-de-saude

13. Brasil. Ministério da Saúde (MS). Secretaria de Atenção em Saúde. Departamento de Atenção Básica. Manual do instrumento de avaliação da atenção primária à saúde: Primary Care Assessment Tool PCATool. Brasília: MS; 2010.

14. Brasil. Ministério da Saúde (MS). Portaria no 3.222, de 10 de dezembro de 2.019. Dispõe sobre os indicadores do pagamento por desempenho, no âmbito do Programa Previne Brasil. Diário Oficial da União; 2019.

15. Harzheim E, Santos CMJ, D'Avila OP, Wollmann L, Pinto LF. Bases para a Reforma da Atenção Primária à Saúde no Brasil em 2019: mudanças estruturantes após 25 anos do Programa de Saúde da Família. Rev Bras Med Família Comunidade 2020; 15(42):2354.

16. Brasil. Instituto Brasileiro de Geografia e Estatística (IBGE). Pesquisa Nacional por Amostra de Domicílios Contínua (PNAD) COVID-19. Rio de Janeiro: IBGE; 2020.

17. Bartoll X, Pérez K, Pasarín M, Rodríguez-Sanz M, Borrell C. Resultats de l'Enquesta de Salut de Barcelona 2016/17 [Internet]. Barcelona: Agència de Salut Pública de Barcelona; 2018 [acessado 2020 Nov 10]. Disponível em: https://www.aspb.cat/wp-content/ uploads/2018/12/ASPB_Enquesta-Salut-Barcelona-2016.pdf

Artigo apresentado em 16/11/2020

Aprovado em 16/11/2020

Versão final apresentada em 18/11/2020

Editores chefes: Maria Cecília de Souza Minayo, Romeu Gomes, Antônio Augusto Moura da Silva 